Titre : Poursuivre le dialogue

Auteur : Paul Bramadat, Julie A. Bettinger et Maryse Guay

Publication : La santé publique à une ère marquée par le doute - Origines religieuses et culturelles de l'hésitation des Canadiens face à la vaccination

Directeurs : Paul Bramadat, Maryse Guay, Julie A. Bettinger et Réal Roy

Pages : 319 - 327

ISBN : 978-2-7622-0359-2

URI : http://hdl.handle.net/11143/16034

DOI : https://doi.org/10.17118/11143/16034

Éditeur : Les Éditions de l'Université de Sherbrooke (ÉDUS) 


\title{
Poursuivre le dialogue
}

\author{
PAUL BRAMADAT, JULIE A. BETTINGER ET MARYSE GUAY
}

\section{Vue d'ensemble}

Lorsque nous avons commencé à discuter de la réticence et de l'opposition à la vaccination au Canada, il y avait de multiples désaccords entre les éditeurs et les auteurs. Après tout, il y a parmi nous des médecins, des infirmières, des humanistes, des experts en sciences sociales et naturelles et des décideurs politiques du pays tout entier, auxquels il faut ajouter des parents, des enfants, des grands-parents, des fratries, des partenaires, des voisins et des amis de gens présentant des attitudes variées au sujet des vaccins. II n'est donc pas étonnant que nos approches, sur ces deux sujets, aient été souvent très différentes. Disons, à tout le moins, que nos conversations ont été plutôt vives. Lors de nos rencontres et de nos discussions, nous nous sommes entendus sur au moins trois points : 1) la réticence et l'opposition à la vaccination lèvent des obstacles complexes et troublants, en ce qui a trait à la santé de notre société; 2 ) les méthodes qu'utilisent les promoteurs de la vaccination ont parfois un effet aliénant sur les membres de sous-cultures particulières; 3 ) les recherches sur ce que nous appelons les motifs religieux et culturels de la réticence à la vaccination ne sont pas encore suffisamment avancées pour que les universitaires, les décideurs et les cliniciens s'en servent comme d'un guide.

Enfait, bien que des reportagessignalant à l'occasion deséclosions de maladies évitables par la vaccination au Canada et à l'étranger nous indiquent des problèmes précis, les universitaires, les cliniciens et le public en général ont très peu de connaissances au sujet de l'importance, de la croissance et des origines de la tendance en faveur de la réticence en général et de ses répercussions'. L'objectif de cet ouvrage ne résidait pas dans la répudiation de la réticence à la vaccination. Après tout, ce phénomène est complexe et ses racines sont nombreuses : culture, religion, interrogations scientifiques légitimes, rumeurs sans fondement, science de pacotille, pensée magique, craintes devant la puissance des grandes sociétés et des gouvernements et simple méconnaissance des faits sous-tendant la vaccinologie et l'épidémiologie. Un seul

1. Pour éviter les longueurs dans le reste de ce chapitre, nous mentionnerons uniquement le concept général de réticence ou d'hésitation face à la vaccination - un spectre dont l'opposition à celle-ci occupe l'une des extrémités. 
ouvrage ne peut - ni ne doit - viser à éliminer la réticence ou à apaiser toutes les inquiétudes au sujet des vaccins. Notre but, dans le cadre de ce projet, était plutôt de donner au lecteur une description générale des forces en présence, selon leur nature (médicale, scientifique, sociale, politique, religieuse et personnelle). Nous espérons que cet ouvrage inspirera nos collègues - et la société en général - à discuter en profondeur des vaccins et de la réticence à les accepter.

Avec cet objectif en tête, les coauteurs ont fait une analyse critique de l'histoire des vaccins et de leur rôle dans la promotion de la santé, ainsi que de leurs manifestations indésirables et de la surveillance de celles-ci; ils se sont également penchés sur la nature des personnes et des groupes qui, pour une ou plusieurs raisons, se méfient des vaccins (ou de la « médecine conventionnelle », en général) et sur les effets de leur réticence, qui affaiblit la protection de l'ensemble des citoyens. Avant de passer aux questions auxquelles d'autres auteurs pourraient porter attention dans l'avenir prochain, il serait utile de résumer certains des principaux arguments présentés dans cet ouvrage.

Premièrement, comme l'ont démontré plusieurs coauteurs, l'hésitation face à la vaccination n'est pas un phénomène nouveau. Depuis la première apparition des vaccins, on s'est toujours interrogé sur leur efficacité, sur le rôle des gouvernements et des fabricants de médicaments dans leur promotion et leur mise au point, sur leur composition et sur le droit que s'arrogent les autorités, les médecins et les pharmaciens d'interférer dans les plans divins ou ceux de la nature. Il y a donc une histoire de la réticence à la vaccination, mais il est également important, pour bien la comprendre, de la considérer comme inévitablement liée à l'action d'autres forces sociales, scientifiques, culturelles et politiques. Selon l'endroit et l'époque, les forces non scientifiques mises en corrélation avec la réticence se révéleront différentes - au siècle précédent, par exemple, les tensions entre les protestants anglophones et les catholiques francophones ont eu un effet sur la perception des vaccins au Québec -, mais certaines, parmi ces différentes forces, semblent perdurer. Il est pertinent de mentionner, dans l'étude des formes actuelles de la réticence, les changements radicaux survenus dans la vie des femmes et les rôles parentaux, la place grandissante que prend Internet comme source de renseignements médicaux courants et le déclin général du prestige et des pouvoirs autrefois conférés aux médecins, hommes et femmes politiques, scientifiques et autres personnages d'influence sur la société.

En deuxième lieu, se faire vacciner ou faire vacciner son enfant est une décision qui comporte des risques, car il arrive que des manifestations indésirables se produisent. Néanmoins, les preuves démontrant que les vaccins prolongent et améliorent la vie au Canada et ailleurs dans le monde sont plus solides que jamais. Par ailleurs, une évaluation non partisane des mécanismes de surveillance de la vaccination actuels, dans de nombreuses sociétés occidentales, devrait inspirer confiance. Ces régimes ne sont évidemment pas infaillibles et ils se distinguent d'une société à l'autre, mais une étude raisonnée des récentes interventions à l'apparition de problèmes d'innocuité montre que les milieux universitaires, les représentants gouvernementaux, les patients et les cliniciens sont en mesure de collaborer afin de rendre les vaccins plus sûrs et d'atténuer les risques qu'ils présentent. Sur la scène publique, les vaccins continuent de susciter la controverse et l'incertitude; la quasi-totalité des scientifiques et des cliniciens, toutefois, conviennent de leur importance fondamentale. On peut voir une analogie intéressante entre la vaccination et les changements climatiques, surtout aux États-Unis, où 
la question soulève des débats interminables dans les médias même après que les experts scientifiques en sont arrivés à un consensus pratiquement total.

Troisièmement, la réticence ou l'opposition à la vaccination est motivée par un grand nombre de raisons. L'utilisation du mot « raisons » peut d'ailleurs porter à confusion. En vérité, une analyse des décisions en matière de santé qui s'articule autour de l'hypothèse selon laquelle elles sont prises après une évaluation rationnelle des possibilités dissimule un autre fait, à savoir que bien des forces déterminant ou influençant nos décisions médicales, telles que les normes sociales, les réactions individuelles et les préférences personnelles, nous sont peu discernables, sinon totalement invisibles, et qu'elles ne sont certainement pas toujours rationnelles. Il est évidemment toujours possible de les connaître, mais seul un excès de naïveté permettrait de supposer que nous puissions les comprendre totalement et encore moins les maîtriser pour en arriver à des décisions parfaitement fondées sur des données probantes. Les valeurs, l'instinct, les émotions, les convictions religieuses et les préjugés cognitifs sont toujours actifs, même si nous désirons en freiner l'intervention au moment de prendre des décisions de santé importantes. C'est pourquoi l'approche reposant uniquement sur le « déficit cognitif » dont souffriraient les Canadiens réticents à la vaccination (selon laquelle le patient ou le parent ne connaît pas l'argument empirique plaidant en faveur des vaccins) est vouée à l'insuccès; la réticence, en effet, n'est pas simplement le résultat d'une erreur cognitive.

En quatrième lieu, on analyse souvent séparément les forces religieuses et culturelles associées à la réticence; on ne se penche sur les premières que lorsqu'un leader ou un groupe religieux conteste la vaccination et on impute surtout aux secondes le recul de la conformité vaccinale. On pourrait en quelque sorte justifier la tendance qui porte à faire une distinction entre, d'un côté, les relativement rares contestations des vaccins ou interrogations à ce sujet provenant officiellement d'institutions religieuses et, de l'autre, les allégations à l'encontre de l'immunisation qui sont tant soit peu liées à des inquiétudes culturelles plus générales portant sur la nature, l'influence des grands fabricants de médicaments, l'autoritarisme gouvernemental et ce que nous pourrions appeler la « chimiophobie ». On peut toutefois aussi se demander s'il existe des points communs entre une bonne partie - mais non la totalité - des types de réticence à la vaccination fondée sur la religion ou la culture. Différents coauteurs du présent ouvrage ont notamment mentionné la ressemblance fréquente entre les caractéristiques «magiques », supra-rationnelles et pourtant intrinsèquement cohérentes d'une réflexion portant sur la religion et la vaccination et le fait qu'elles s'opposent de manière pratiquement similaire au consensus de la science conventionnelle.

Cinquièmement, cet ouvrage présente, parmi ses arguments fondamentaux, le fait qu'il est non seulement possible, mais nécessaire d'entamer un dialogue signifiant avec les parents ou patients qui sont réticents ou s'opposent à la vaccination. Au début, certains de nos collègues doutaient qu'une interaction avec des personnes souvent hostiles à la science ou à la médecine ou ayant des opinions extravagantes à ce sujet puisse être fructueuse. Observons en passant que les spécialistes des religions ont démontré la très grande rareté d'une interruption totale de la communication entre les individus. En fait, il est très fréquent que les gens passent tour à tour d'une conversation sur des allégations empiriquement vérifiables à une autre sur des intuitions stimulantes sur le plan émotif ou encore à une autre portant sur des sensibilités religieuses incontournables ou inspirées par elles. Non seulement discutons-nous de ces manières différentes avec nos voisins, collègues ou amis, mais on peut aussi dire que dans chacun d'entre nous, à des degrés divers, ces sensibilités intellectuelles et épistémologiques 
coexistent (souvent sans que nous ayons l'impression, pour ainsi dire, de changer de registre). Ces explications ne signifient pas, évidemment, que les échanges entre les partisans et les critiques de la vaccination se feront sans irritation, ni rancœur. Néanmoins, une connaissance de ce que nous pourrions appeler la diversité cognitive sociale et personnelle nous remet à notre place et pourrait nous aider à accepter la critique de manière plus productive.

Enfin, plusieurs auteurs ont constaté que les écrits scientifiques et la pratique clinique confirment tous deux que le meilleur moyen d'encourager les parents et les patients à envisager sérieusement la vaccination consiste, pour les partisans de celle-ci, à nouer avec eux des relations à long terme. Bien qu'il soit important de faire connaître le consensus médical entourant la vaccination (y compris ses risques) aux personnes, familles et groupes réticents, on ne peut s'attendre, sans faire preuve d'une certaine condescendance, à ce que tous les patients et parents mettent de côté leurs inquiétudes immédiatement. Dans ce cas comme dans celui du dialogue entre croyants de collectivités religieuses radicalement différentes (de même qu'entre croyants et athées), le temps investi rapporte de plus grands dividendes, à l'échelle de la société, que les débats obstinés au sujet de convictions théologiques divergentes, où il n'y a qu'un seul vainqueur.

\section{Prochaines étapes}

Et maintenant, que nous réserve l'avenir? Cet ouvrage contribuera grandement au dialogue qui se tient au Canada au sujet de la vaccination. II faut cependant, pour que ce dialogue se poursuive, investir encore plus en temps et en ressources. Nous indiquons, dans les paragraphes qui suivent, les sujets les plus criants, sur le plan des systèmes et de la culture, vers lesquels les chefs de gouvernement, les chercheurs, les prestataires de soins de santé et les professionnels de santé publique doivent diriger leur attention.

Les données et les théories avancées dans ces pages suggèrent notamment que les administrations locales et les gouvernements provinciaux et fédéral doivent appuyer la vaccination par différents moyens (financement, éducation, recherche et politiques). En 2013, le Canada se classait avant-dernier, parmi les 29 pays développés, pour la couverture vaccinale (UNICEF, 2013). Cette situation est plutôt inquiétante. Il est essentiel d'en arriver à évaluer la couverture et à la suivre de près de manière régulière. En particulier, on doit veiller à ce que les treize provinces et territoires adoptent un calendrier d'immunisation national et standardisent les programmes à l'échelle du pays, sur la base de données scientifiques probantes de la plus grande qualité, ce qui serait bien accueilli par tous les praticiens de santé publique et les prestataires de soins II faut reconnaître que l'élaboration d'une stratégie nationale s'est butée au principe fortement enraciné conférant l'autonomie aux gouvernements provinciaux et territoriaux en matière de programmes de santé, mais il est tout de même difficile d'accepter les très importantes différences entre les calendriers d'immunisation à l'échelle nationale, car elles engendrent la confusion chez les parents et les prestataires de soins de santé et laissent supposer, par inadvertance, qu'on ne s'entend guère sur l'importance de la vaccination. 
Une méthode simple pour normaliser les approches en matière de vaccination - et d'en assurer un suivi plus efficace - serait de faire en sorte que tous les territoires et provinces établissent des registres d'immunisation électroniques. Les dossiers d'immunisation personnels doivent être transmissibles et disponibles autant pour le vacciné que pour tous les professionnels de la santé, à de multiples établissements. Les registres devraient en outre permettre d'évaluer de manière fiable la couverture vaccinale au sein de la population. Comme les vaccins sont dispensés de différentes façons, par différents professionnels (médecins, infirmières, etc.) et dans des lieux variés (écoles, cabinets de médecin, pharmacies, milieux de travail, etc.), nous devons élargir de beaucoup la portée de nos registres d'immunisation actuels ${ }^{2}$.

En dernier lieu, en matière de politique, nos gouvernants et les autorités en santé publique devraient offrir une contrepartie financière aux rares personnes à qui un vaccin cause du tort. Il existe déjà des programmes de compensation de ce genre aux États-Unis et au Québec; il est temps que le reste du pays leur emboîte le pas. Si nous demandons aux citoyens de se faire vacciner au titre du "bien commun », nous devons venir en aide à ceux qui, rarement mais bien réellement, sont affligés d'une manifestation indésirable qui en découle. Tant que nous demeurerons incapables de régler ce problème collectivement, les indécis disposeront encore d'une objection solide et légitime contre la vaccination.

Au-delà des enjeux systémiques et politiques, il faut absolument en arriver à mieux comprendre les facteurs individuels et culturels qui motivent l'hésitation face à la vaccination. Les auteurs du présent ouvrage ont particulièrement reconnu la pertinence d'une étude de l'évolution rapide et radicale de la religion au Canada. Compte tenu de l'accroissement du nombre des fidèles de groupes religieux principalement non occidentaux (musulmans, hindous, sikhs, bouddhistes, etc.) et de l'arrivée relativement récente d'immigrants d'obédience chrétienne de sociétés autres qu'européennes, les peofessionnels de soins la santé devront imaginer d'autres moyens de promouvoir la vaccination au sein de la société canadienne. La plupart du temps, les immigrants sont en faveur de la vaccination, mais les défenseurs de celle-ci devront être sensibilisés aux questions sociales, morales, religieuses et politiques qui intéressent ces groupes, afin de prévenir les mésententes susceptibles de se développer à partir de rumeurs (et qui pourraient s'envenimer plus facilement parmi ceux qui ne sont pas encore totalement intégrés dans la société canadienne) et celles que pourraient susciter de nouveaux vaccins comme celui qui prévient l'infection par le VPH (et qui souvent crée une controverse sur l'activité sexuelle des jeunes et le contrôle parental).

Il faut également porter attention aux formes fondamentalistes du christianisme, qui survivent et se renforcent même dans des régions généralement acquises au libéralisme non confessionnel. Des travaux de recherche en ethnographie pourraient notamment contribuer à la détermination de méthodes favorisant la création de relations constructives et durables

\footnotetext{
2. Bien que, dans le cadre de registres comme le registre canadien Panorama, certaines données sont accessibles aux autorités de santé publique, il est peu probable qu'ils contiennent une information sur la population au complet et ils demeureront inaccessibles à de nombreux prestataires désireux de vérifier le dossier de vaccination de patients donnés. Plusieurs provinces ont en outre décidé de se retirer de Panorama, ce qui rendra les comparaisons à l'échelle nationale difficiles, sinon impossibles. Des applications mobiles, telles que http://www. immunize.ca, transmettent un dossier électronique sur un téléphone intelligent et permettent au moins à son possesseur de rechercher les données qui lui sont propres et, éventuellement, de les remettre à un prestataire de soins de santé. Une solution de cette nature ne satisfait cependant pas aux exigences de la surveillance en santé publique, ni ne procure des estimations suffisantes sur la couverture d'une population. II faudrait trouver un mécanisme regroupant l'application personnelle et une base de données relevant de la santé publique.
} 
entre la collectivité libérale dominante (sans doute partisane de la médecine traditionnelle) et les groupes chrétiens évangéliques plutôt isolés. Cela permettrait probablement de réduire les éclosions de maladies du genre de celles qui sont notamment survenues récemment dans différentes régions de la Colombie-Britannique. En plus d'enrichir la recherche ethnographique visant les personnes et les groupes réticents à la vaccination pour des motifs religieux, il faudrait aussi étudier plus exhaustivement leurs théologies, philosophies, théodicées (qui expliquent les motifs de la souffrance humaine) et modes de réflexion. Comme nous l'avons mentionné précédemment, la plupart des gens vivent sans réflexion systématique constante; par conséquent, une compréhension des racines théologiques de l'existence de leur collectivité ne constituerait pas une méthode simple pour en arriver à répondre à leurs interrogations. Par contre, une certaine connaissance des principes intellectuels et spirituels en jeu permettrait peut-être de combler l'écart entre les groupes réticents et les partisans de la vaccination.

Par ailleurs, il est essentiel de prendre en considération les liens qui existent entre la progression de la réticence dans notre société et la croissance extrêmement rapide d'une cohorte qui n'a que faire des institutions religieuses, mais persiste à adopter diverses visions de l'univers qui sont clairement rattachées à des sensibilités spirituelles variées. Bon nombre des personnes et des groupes dont il a été question dans cet ouvrage - et particulièrement ceux qui sont nés depuis la fin des années 1960 - se décrivent comme étant préoccupés de spiritualité, mais non religieux. Bien qu'ils ne soient pas des membres officiellement actifs ou des fidèles exclusifs d'une religion traditionnelle, sous quelque forme qu'elle se présente, leur approche vis-à-vis de la nature, du cosmos, du capitalisme, de l'autonomie personnelle, de la biomédecine, des médecines alternatives et de leur propre santé est clairement en partie inspirée par la spiritualité. C'est pourquoi il est approprié, voire probablement nécessaire d'appliquer à leur égard les outils d'analyse que l'on utilise dans l'étude d'autres formes de religion ou de spiritualité.

On peut avoir recours, sur place, à des méthodes quantitatives et qualitatives pour décrire les communautés, groupes et individus réticents à la vaccination, y compris les professionnels de la santé, et en faire l'interprétation. Il faut pour cela éduquer et former ces derniers et intégrer, dans le travail sur le terrain et les activités de mobilisation, ceux d'entre eux qui demeurent indécis. On pourrait également repérer les groupes à risque grâce à des travaux de cartographie permettant de prévoir où auront lieu de prochaines éclosions, sur la base de l'évolution des données démographiques fondées sur la religion. Les travaux qui suivent le développement de nouveaux discours culturels sur la science, la nature, le gouvernement et le corps humain pourraient saisir les tendances récentes et prévoir une intervention, éventuellement avant que de telles réorientations culturelles n'influencent l'acceptation de la vaccination. Ils créeront ainsi des occasions de nouer des relations avec ces groupes et de rechercher de concert des solutions respectueuses des exigences de santé publique, de l'autonomie individuelle et des convictions et pratiques religieuses et spirituelles. Des interactions personnelles régulières entre les personnes réticentes, les cliniciens et les universitaires ayant déjà étudié les discours traitant de la religion et de la santé pourraient permettre de découvrir des méthodes acceptables sur le plan religieux et culturel de promouvoir la vaccination ou, à défaut, d'autres moyens de contrôler la maladie. 
Enfin, nous ne pouvons négliger la nécessité de mieux comprendre la réticence à la vaccination à l'échelle internationale et de réaliser des études pour examiner comment on y réagit dans d'autres pays (Larson, Jarrett et coll., 2014).

Selon de nombreux coauteurs du présent ouvrage, ce phénomène ne se manifeste pas qu'au Canada; en vérité, nombreux sont les discours sous-tendant la réticence à la vaccination dont la source et même la médiation sont étrangères. Il est important, à ce stade-ci, de se rappeler quel rôle important joue Internet dans la progression du mouvement de réticence et dans un éventuel mouvement contraire (Larson, Wilson et coll., 2014). II est devenu inutile de faire le décompte des histoires, anecdotes, théories du complot, demi-vérités et vérités qui se retrouvent simultanément dans les pages Web consacrées à la vaccination, maintenant assez nombreuses. Les discours en question plongent néanmoins leurs racines dans des groupes très particuliers; ils les influencent de manière disproportionnée, mais on ne peut les comprendre en faisant abstraction de la nature de ces groupes. La perspective donnant une vue appropriée de la réticence à la vaccination est donc à la fois internationale et locale. Heureusement, on est de plus en plus sensibilisé à ce phénomène. En 2012, l'Organisation mondiale de la Santé (OMS) a même formé un groupe de travail appelé le Groupe stratégique consultatif d'experts (SAGE), qui a pour tâche de définir la réticence à la vaccination et sa portée (OMS, 2014, 7) et de l'étudier dans différents contextes (14). Parmi ses membres figurent deux expertes d'origine canadienne ayant collaboré au présent ouvrage (Eve Dubé et Noni MacDonald). Le groupe a publié en 2014 un rapport qui donnait une définition de l'hésitation face à la vaccination et cernait les travaux de recherche à exécuter et les stratégies à adopter pour la circonscrire. On indiquait notamment la nécessité d'approfondir l'étude des facteurs culturels et religieux contribuant à ce mouvement à l'échelle internationale.

Même si les autorités médicales et politiques n'ont plus autant d'influence qu'auparavant, leur participation active dans le dialogue concernant la réticence à la vaccination est critique si les progrès réalisés jusqu'ici en santé publique au Canada et ailleurs dans le monde doivent se maintenir. Même si, depuis au-delà d'un siècle, on a la preuve que les vaccins sont un outil essentiel en santé publique, l'indécision à ce sujet prend de l'ampleur dans de nombreuses sociétés. C'est pourquoi nous avons atteint, dans notre histoire, une étape déterminante, qui exige réflexion. La réticence à la vaccination présente maintenant un grand dilemme en santé publique et la meilleure façon de le résoudre, pour nous tous, consiste à attirer la participation d'autant de voix que possible dans le débat public qu'elle suscite toujours et à faire progresser la recherche en santé publique et en vaccinologie. Les auteurs et les éditeurs du présent ouvrage ont voulu participer aux débats que soulève la vaccination dans notre société et créer un contexte dans lequel la réticence à la vaccination, sous ses différentes formes complexes et d'après les histoires qu'elle comporte, puisse faire l'objet de délibérations ouvertes, faisant une place à la critique, mais empreintes de respect mutuel. Nous espérons que ces pages marqueront le début d'un dialogue aussi durable que fructueux. 


\section{BIBLIOGRAPHIE}

Larson, H.J., C. Jarrett, E. Eckersberger, D.M. Smith, and P. Paterson. 2014. "Understanding Vaccine Hesitancy around Vaccines and Vaccination from a Global Perspective: A Systematic Review of Published Literature, 2007-2012." Vaccine 32 (19): 2150-9. http://dx.doi.org/10.1016/j.vaccine.2014.01 .081.

Larson, H.J., R. Wilson, S. Hanley, and P. Paterson. 2014. "Tracking the Global Spread of Vaccine Sentiments: The Global Response to Japan's Suspension of Its HPV Vaccine Recommendation." Human Vaccines \& Immunotherapeutics 10 (9): 2543-50. http://dx.doi.org/10.4161/21645515.2014.969618.

UNICEF. 2013. Innocenti Report Card 77: Child Well-Being in Rich Countries: A Comparative Overview. April. Florence: UNICEF Office of Research. https://www.unicef-irc.org/publications/pdf/rc11_eng.pdf.

World Health Organization (WHO). 2014. Report of the SAGE Working Group on Vaccine Hesitancy. 1 October. Geneva. http://www.who.int/immunization/sage/meetings/2014/october/1_Report_WORKING_GROUP_vaccine_hesitancy_final.pdf. 